\title{
Radiation associated coronary occlusion: Overlooked as trastuzumab-induced cardiomyopathy
}

\author{
Ugur Turk $^{1^{*}}$, Veysel Yavuz ${ }^{2}$, Nurullah Cetin ${ }^{2}$ \\ ${ }^{1}$ Department of Cardiology, Central Hospital, Izmir, Turkey \\ ${ }^{2}$ Department of Cardiology, Celal Bayar University, Manisa, Turkey \\ Email: droturk@yahoo.com, veyselyavuz@yahoo.com,nurullah_ctn@hotmail.com
}

Received 15 January 2012; revised 25 February 2012; accepted 6 March 2012

\begin{abstract}
Trastuzumab is an agent used for receptor-positive breast cancer. Up to a third of patients treated with trastuzumab might develop cardiomyopathy. Many patients with breast cancer have taken pre- or co-administration of mediastinal radiotherapy. Both of treatment modalities have toxic effects on myocardium. It may result with diagnostic confusion between radiation-induced CAD (riCAD) and trastuzumab cardiomyopathy in the case with dilated cardiomyopathy. TTE may offer clues of differential diagnosis.
\end{abstract}

Keywords: Cardiomyopathy; Trastuzumab; Radiation

\section{INTRODUCTION}

Radiation injury to the heart includes constrictive pericarditis, myocardial fibrosis, valvular and coronary artery lesions. Higher risk of coronary artery disease (CAD) has been reported for patients treated with mediastinal irradiation. [1]

Trastuzumab is used for receptor-positive breast cancer. Up to a third of patients treated with trastuzumab might develop cardiomyopathy. Transthoracic echocardiography (TTE) is first diagnostic tool of these patients [2]. TTE may offer clues of differential diagnosis in the case of diagnostic confusion between radiation-induced CAD (riCAD) and trastuzumab cardiomyopathy.

Herein, we reported the case of riCAD who had diagnosed as trastuzumab cardiomyopathy before.

\section{CASE}

A 47-year-old woman presented with dyspnea. The patient had history of ductal carcinoma of left breast in 2003. Same year she underwent radiotherapy followed by chemotherapy. Right breast metastasis was found 2 year before and she received trastuzumab since 6 months before presentation.

\footnotetext{
${ }^{*}$ Corresponding author.
}

At presentation, physical examination was unremarkable. Laboratory data including cardiac troponin $\mathrm{T}$ were normal. ECG demonstrated loss of R waves in leads V1 to V4. TTE revealed global hypokinesia of left ventricle (LV). The diagnosis was trastuzumab cardiomyopathy based on symptoms and TTE findings. Three months later, follow-up TTE revealed hypokinesias of LV apical and anterior segments. Retrospective analysis of previous TTE demonstrated same findings with last TTE. Coronary angiography was planned. It revealed occlusion in distal segment of the left anterior descending artery (LAD) (Figure 1, arrows) and akinetic LV apex (Figure 2, dashed line represents diastolic border). There were noany coronary lesion and/or stenosis except the LAD occlusion. Evaluation of myocardial viability with Thallium-201 SPECT showed no viability and conservative

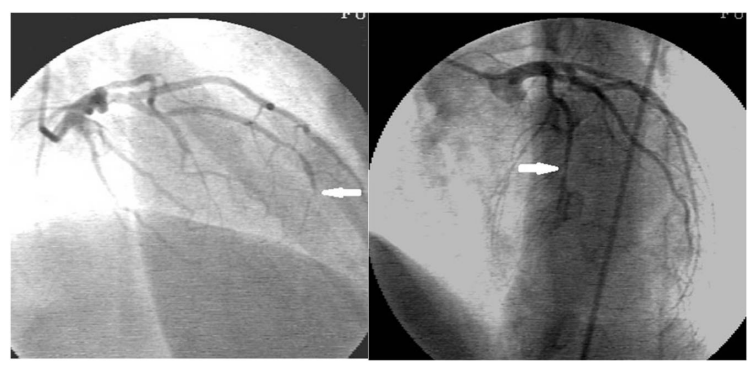

Figure 1. Occlusion in distal segment of the left anterior descending artery (arrows).

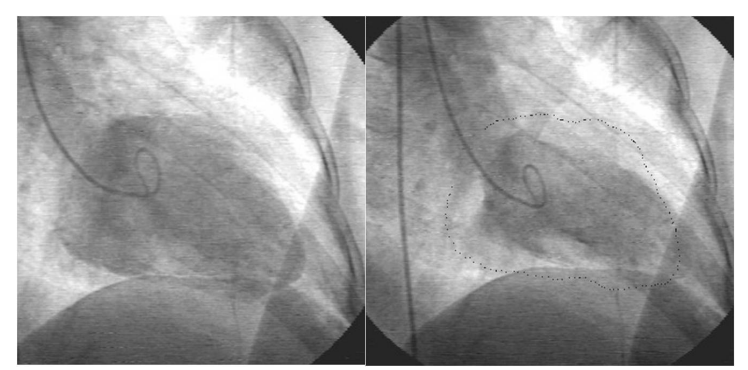

Figure 2. Left Ventriculography showed akinetic LV apex. Left; End-diastolic frame. Right; End-systolic frame. Dashed line represents end-diastolic border. 
management was decided.

\section{DISCUSSION}

CAD can be reasonably ascribed to the effects of chest irradiation when the patients are young and free from risk factors [1]. The mechanism responsible for riCAD is not clearly defined but is thought to relate to damage to the coronary endothelium, leading to significant fibrosis. LAD is the most frequent artery involved in riCAD [3]. riCAD rarely occurs within the first 10 years after exposure and characteristically affects ostial and proximal segments of coronary arteries [3].

Lacks of CAD risk factors, early presentation of CAD, atypical coronary localization for atherosclerotic CAD were suggestive features of riCAD in our patient. However, atypical clinical characteristics for riCAD in our patient were no angiographic evidences of CAD at ostial and proximal coronary segments and relatively early presentation after exposure (8 years).

Another clinical challenge in our patient was making the differential diagnosis between riCAD and trastuzumab induced cardiomyopathy. TTE evaluation of the patient offered clues for solving the problem. Demonstration of regional wall motion abnormalities of LV unlike from global wall motion abnormalities on TTE reflects a specific coronary lesion. Regional hypokinesia suggested CAD evolving LAD in our patient. However globally hypokinetic LV may result of trastuzumab cardiomyopathy or radiation induced myocardial fibrosis. This case highlights the importance of TTE in the cases of drug/radiation induced cardiotoxicity. Thus, clinicians should mention the history of mediastinal radiotherapy while referring a patient to an echocardiographer for the evaluation of a drug induced cardiotoxicity.

\section{REFERENCES}

[1] Roychoudhuri, R., Robinson, D., Putcha, V., et al. (2007) Increased cardiovascular mortality more than fifteen years after radiotherapy for breast cancer: A population-based study. BMC Cancer, 7, 9-14.

[2] Guglin, M., Hartlage, G., Reynolds, C., et al. (2009) Trastuzumab-induced cardiomyopathy: Not as benign as it looks? A retrospective study. Journal of Cardiac Failure, 15, 651-657.

[3] Feng, M., Moran, J.M., Koelling, T., et al. (2011) Development and validation of a heart atlas to study cardiac exposure to radiation following treatment for breast cancer. International Journal of Radiation Oncology, Biology, Physics, 79, 10-18. 